\title{
Emergency Surgical Repair of Post Infarction Ventricular Septal Rupture Case Report
}

\author{
Yasser Mubarak ${ }^{1}$ \\ ${ }^{1}$ Minia University Faculty of Medicine
}

November 19, 2020

\begin{abstract}
Ventricular septal rupture (VSR) is a life-threatening complication of trans-mural acute myocardial infarction (MI). Surgical intervention remains the treatment of choice, but it is still a challenging operation associated with high mortality (1). VSR is a rare serious complication of MI. Its incidence has been estimated between $1 \%\urcorner-2 \%$ after MI. If it treated medically the mortality is very high ( $25 \%$ in 1 st day, $75 \%$ at 1 st week and $90 \%$ at 2 months). Patients that survive following medical treatment usually have clinically poor cardiac function. So, the result of medical treatment of VSR is disappointing (2). Primary PCI following MI can be significantly reduced the incidence of mechanical complications. However, with improving treatment, appropriate management of mechanical complications remains a cornerstone to avoid low cardiac output (LCO) and multi organ failure (MOF). Medical treatment has high failure and mortality rate, and early surgical ventricular septal defect (VSD) closure is recommended to reverse the hemodynamic deterioration. However, surgical repair is associated to a high rate of mortality $(20 \%-40 \%)$ even in the more recent reports (3). Transcatheter device closure of VSR, is an option, however it is generally accepted to be inadequate. Progressive deterioration in hemodynamic status makes surgical intervention often the only realistic option (4).
\end{abstract}

\section{Emergency Surgical Repair of Post Infarction Ventricular Septal Rupture}

\section{Case Report}

Yasser Shaban Mubarak, MD.

Assistant Professor of Cardiothoracic Surgery Department, Faculty of Medicine, Minia University, Egypt.

Associated Consultant adult cardiac surgery at Madinah Cardiac Center, King Fahad Hospital, Madinah, KSA

Corresponding author: Yasser Shaban Mubarak. E-mail: yassermubarak73@gmail.com.

Tel: +966560708223.Cardiothoracic surgery Department. Minia University. ORCID 0000-0001-8460-2

Availability of data and material: - It is available from recording files and data at cardiothoracic surgery departments and cardiology clinics for follow up.

Competing interest: None to declare

Funding: None

Introduction

There has been a reduction in the incidence of post MI VSR because of primary PCI. Anterior MI is more likely to cause apical or anterior septal defects, and inferior or lateral MI is more likely to cause basal or posterior septal defects (1) . 
The incidence of post-infarction VSR is reported as 1-2\% after AMI. However, the incidence may be recently decreased with early interventions after AMI including thrombolytic therapy, primary PCI, and urgent CABG. The timing to develop post-MI VSD is variable, in a few hours up to 2 weeks (average time of 2-4 days). It is usually associated with complete occlusion of a single coronary artery with poor collateral vessels. About $2 / 3$ of post-infarction VSDs are located in the antero-apical septum, while the remaining in posterior septum, from left anterior artery (LAD) and dominant right coronary artery (RCA) or circumflex artery $(\mathrm{Cx})$ occlusions. The natural history of untreated VSR is extremely poor. The early development of congestive heart failure (CHF) leading to cardiogenic shock is the primary cause of death. Ventricular dysfunction may be left or right, depending on the territory infracted and the location of VSR. Especially in posterior infarct, significant mitral valve regurgitation (MR) can play an important role in the development of heart failure, resulting in elevated left ventricular end-diastolic pressure (LVEDP) and pulmonary edema. The degree of left-to-right shunt depends on the size of the VSD and pressure gradient across the septum. If the shunt is large, the normally compliant right ventricle (RV) will not tolerate the sudden increase in load, and the development of significant RV failure. As a result, severe biventricular failure is not uncommon in patients with post-infarction VS (5) .

\section{Case report}

Ethics approval and consent to participate that have read and understood the information about the research as provided in the participant information sheet inside his file. The study has got the formal approval and permission from before to start the study. The study conformed to the principles of "Declaration of Helsinki" and the investigator followed the appropriate safeguards regarding the rights and welfare of the human participants that have been included in the performed study. The principle investigator has got the formal approval and permission from the relevant Cardiac Center Heart Team prior to commencement of the study. Consent for publication was obtained written consent from patients.

A 49 year male with complaint of sudden onset chest pain was referred to cardiac center. The patient was found to have acute inferior ST- segment elevation (STEMI). He denied a history of angina or myocardial infarction. The patient was a heavy smoker, diabetes mellitus (type I) with a family history of coronary heart disease (CAD). Coronary angiography was showed total occluded paraosteal right coronary artery (RCA), and subsequently underwent percutaneous coronary intervention (PCI). Bedside Trans Thoracic Echography (TTE) demonstrated a VSR 15mm located basal inferoseptal at level of mitral valve, moderate to severe mitral regurgitation (MR), severe tricuspid regurgitation (TR), and biventricular dysfunction (EF <30\%) [Fig.1] . Patient was in cardiogenic shock and pulmonary edema, an intra-aortic balloon pump (IABP) was inserted for pressure support. On physical examination, the patient was restless and in overt distress, and a pansystolic murmur was auscultated from the left sternal border. The blood pressure was $80 / 50 \mathrm{~mm} \mathrm{Hg}$, pulse $125 \mathrm{bpm}$, respiratory rate $24 \mathrm{bpm}$. His skin was cool and poorly perfused with shallow respiration, and weak peripheral pulses. Laboratory investigations were showing troponin $0.170 \mathrm{ng} / \mathrm{mL}$, serum creatinine level of $1.3 \mathrm{mg} / \mathrm{dL}$, and MB-CK level of $229 \mathrm{U} / \mathrm{L}$. Chest X-ray showed increased cardiothoracic ratio with remarkable lung congestion [Fig.2], and ECG showed sinus rhythm and STEMI in leads II, III, and aVF. Post-infarction posterior VSR was diagnosed, and emergency surgery was decided. Medical management is aimed to improve cardiac output and reduce the left to right shunt by reducing systemic vascular resistance and LV pressure. Inotropes, diuretics, and placement of IABP are often used.

Emergency transferred to operative room (OR) for VSD repair, because cardiogenic shock in the setting of post infarction VSD is a surgical emergency. Median sternotomy, then cardiopulmonary bypass (CPB) with moderate hypothermia $\left(32^{\circ} \mathrm{C}\right)$ was established between both caval veins with caval tapes and ascending aorta. An aortic cross-clamp was placed, and antegrade blood cardioplegic arrest was induced. Deep left pericardial traction sutures were placed to facilitate exposure of the posterior wall of the heart. We made longitudinal incision lateral and parallel to posterior descending artery (PDA) and identified a VSR in the middle level of the posterior wall. Stay sutures were placed to expose the edges of the defect and to help visualize the interior of the ventricle. Exposure The necrotic myocardium around the VSR was resected, revealing a defect measuring $2 \mathrm{~cm}$ in diameter. Closure of VSR without tension employed using a bovine 
pericardial patch placed on the LV side of the septum, and Teflon felt pledgets placed on the RV and RV free wall. The edges of the ventriculotomy are re-approximated with a double layer buttressed closure with Teflon felt. Biological glue is often used to ensure complete hemostasis. [Fig.3-4] Postoperative TTE revealed no residual shunt, EF 35\%, mild MR, and moderate TR. Patient was discharged from hospital on $15^{\text {th }}$ POD.

\section{Discussion}

Patient's myocardium with post-infarction VSD is characterized by severe dysfunction. The "additive ischemia" caused by aortic cross clamp, ischemia and reperfusion injury, has a significant aggravation to the myocardium and overall negative impact to patient's outcome(6). So, the operations are very high risk, and try to do the best during myocardial preservation.

Post infarction VSR constitutes a severe mechanical complication of CAD with very high surgical morbidity and mortality up to 50\%. Many factors contribute to an unfavorable surgical outcome such as the emergency, presence of 3-MVD, posterior VSR, incomplete revascularization, intractable shock and MOF. The adequate myocardial protection during the operation is considered to be the cornerstone for a better outcome postoperatively (6). In our case, the risk factors were emergency, cardiogenic shock, and posterior VSR.

Preoperative cardiogenic shock and early post infarction VSR carry a grave prognosis. Achieving hemodynamic stability prior to surgery may be beneficial but prolonged attempts to improve patients' cardiovascular state are hazardous (2). Preoperative maximized inotropics support and placement of IABP was essential to decrease left to right shunt, and MOF.

Repair of post infarction VSD is still a challenging procedure with a high risk of residual shunt and high mortality. CABG can be done safely to control the added risk of an associated CAD, especially MVD should be routinely revascularised (3-4). The role of primary PCI or concomitant CABG in the setting of VSR closure is debatable as myocardial damage is already transmural and residual viability therefore questionable. However, PCI was done previous to RCA.

\section{Conclusion}

Post infarction VSD has high mortality. Emergency Surgical closure is mandatory after optimized medical and mechanical support. Revascularization is still challenged because viability is not convinced.

\section{Abbreviations}

VSR: ventricular septal rupture, VSD: ventricular septal defect, AMI: acute myocardial infarction,STEMI: ST segment elevation MI, CAD: coronary artery disease, CABG: coronary artery bypass grafting, PCI:percutaneous coronary intervention, IABP: intra-aortic balloon pump, LAD: left anterior descending artery, CX:circumflex, RCA: right coronary artery, PDA: posterior descending artery, MVD: multivessel disease TTE: trans thoracic echo, CPB: cardiopulmonary bypass EF:ejection fraction, CHF: congestive heart failure, LCO:low cardiac output, MOF: multi organ failure, MR:mitral regurgitation, TR: tricuspid regurgitation LV:left ventricle, RV: right ventricle, LVEDP: left ventricular end diastolic pressure, POD: post-operative day, OR: operative room.

\section{References}

1-Furukawa K, Shirasaki Y, Ishii H, Nakamura E, Nakamura K.Extended sandwich technique via the right atrial approach for post-infarction posterior ventricular septal rupture. General Thorac Cardiovasc Surge. 2020; 68:629-32.

2-Deja MA, Szostek J, Widenka K, Szafron B, Spyt TJ, Hickey M, et al. Post infarction ventricular septal defect can we do better? Euro J Cardiothorac Surg. 2000; 18: 194-201.

3-Labroussea L, Choukrouna E, Chevalierb JM, Madonnaa F, Robertiea F, Merlicoa F, et al - Surgery for post infarction ventricular septal defect (VSD): risk factors for hospital death and long term results. Euro J Cardiothorac Surg. 2002; 21: 725-32. 
4-Barkera TA, Ramnarineb IR, Wood EB, Grayson AD, Aud J, Fabri BM, et al . Repair of post-infarct ventricular septal defect with or without coronary artery bypass grafting in the northwest of England: a 5-year multi-institutional experience. Euro J Cardiothorac Surg. 2003; 24: 940-46.

5-Gilmanov D, Farneti P, Solinas M, Glauber M. Severe mitral regurgitation in patients with postinfarction inter-ventricular septal defect: a simple way of simultaneous valve repair. Euro J Cardio-Thorac Surg. 2013; 43: 184-86.

6-Apostolakis E, Kallikourdis A, Baikoussis NG, Dedeilias P, Dougeni D. The non-ischemic repair as a safe alternative method for repair of anterior post-infarction VSD Journal of Cardiothoracic Surgery 2010, 5:6. doi:10.1186/1749-8090-5-6

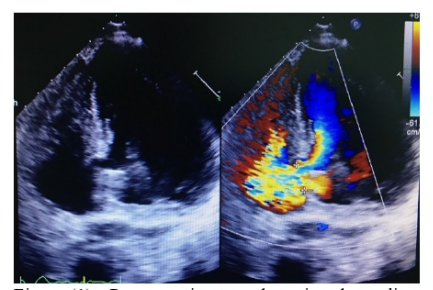

Figure (1):- Pre-operative transthoracic echocardiogram shows a ventricular septal rupture with left-to right shunt flow

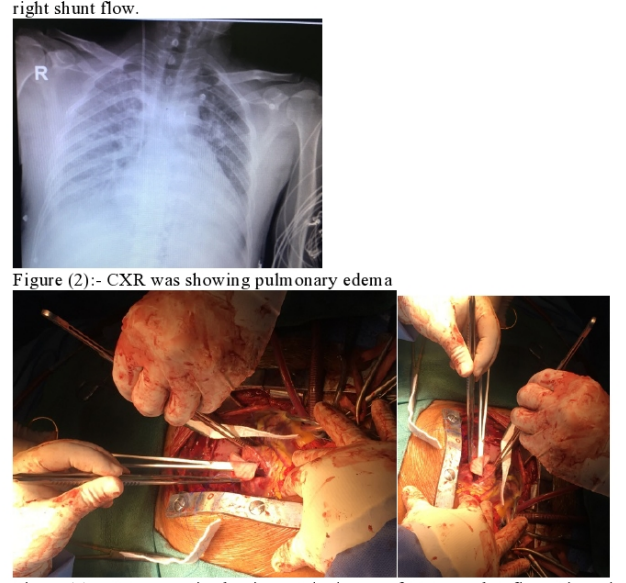

Figure (3): - Intraoperative bovine patch closure of VSR, and Teflon strips reinforced linea ventriculotomy closure.

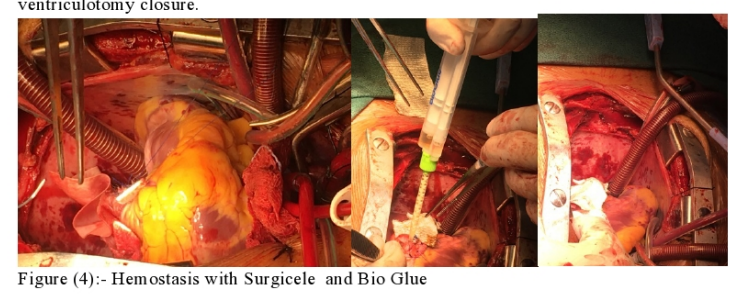

